Disponível em:

http://editora.unoesc.edu.br/index.php/race

RACE, Joaçaba, v. 17, n. 2, p. 567-590, maio/ago. 2018

\title{
A INFLUÊNCIA DA EDUCAÇÃO FINANCEIRA NA INSERÇÃO DOS INVESTIDORES NO MERCADO DE CAPITAIS BRASILEIRO: UM ESTUDO COM DISCENTES DA ÁREA DE NEGÓCIOS
}

The influence of financial education in integration of investors in Brazil's capitals market: a study with students of the business area

Klerton Andrade Freitas de Amorim

E-mail: klertonandrade@gmail.com

Graduado em Contabilidade pela Universidade Federal da Paraíba; Extensão acadêmica em Avaliação Econômica de Projetos Sociais pela Fundação Itaú Social; Professor de Investimentos na SIR Educação Financeira; Assessor de Investimentos credenciado à XP Investimentos. Endereço para contato: Avenida Nego, 270, Tambaú, 58039-100, João Pessoa, Paraíba, Brasil.

Greicy Kelly Farias Lucena E-mail: greicy.lucena49@gmail.com Graduanda no Curso de Ciências Contábeis da Universidade Federal da Paraíba; Pesquisadora colaboradora no Núcleo de Pesquisas em Educação Financeira e Imóveis do Instituto Federal da Paraíba; Trainee de Contabilidade na Central Sicredi NNE.

\section{Luiz Felipe de Araújo Pontes Girão}

E-mail: LFAPG@hotmail.com

Doutor em Contabilidade pela Universidade de Brasília (UnB/UFPB/UFRN); Mestre pela Universidade de Brasília (UnB/UFPB/UFRN); Professor Adjunto I no Departamento de Finanças e Contabilidade, nos cursos de graduação e pós-graduação da Universidade Federal da Paraíba.

Dimas Barrêto de Queiroz

E-mail: dimasqueiroz@gmail.com

Doutor em Ciências Contábeis pelo Programa Multi-institucional e Inter-Regional de Pós-graduação em Ciências Contábeis pela Universidade de Brasília (UnB/ UFPB/UFRN); Mestre em Ciências Contábeis pelo Programa Multi-institucional e Inter-Regional de Pós-graduação em Ciências Contábeis pela Universidade de Brasília (UnB/UFPB/UFRN); Professor Adjunto II no Departamento de Finanças e Contabilidade e membro do corpo docente permanente do Programa de Pós-graduação em Ciências Contábeis da Universidade Federal da Paraíba.

Artigo recebido em 31 de março de 2018. Aceito em 24 de junho de 2018. 


\section{Resumo}

A educação financeira e o entendimento do mercado de capitais abrem as portas de interação entre os agentes econômicos. Autores afirmam que o funcionamento do mercado de capitais no Brasil é conhecido por poucos, contudo, ressaltam que apesar desses aspectos, parece existir uma preocupação recente em atrair os investidores com credibilidade e sustentabilidade. Nesse sentido, no presente estudo investigou-se o nível de educação financeira de discentes da área de negócios de uma universidade pública e a sua relação com a participação no mercado de capitais. Para tal fim, definiu-se a hipótese de que a educação financeira dos investidores influencia positivamente a probabilidade de participação no mercado de capitais brasileiro. Para a elaboração do indicador de educação financeira, coletaram-se os dados necessários baseados nos quesitos utilizados por Atkinson e Messy (2012), publicados na Organization for Economic Cooperation and Development (OECD). O método utilizado foi a regressão logística múltipla, cujo principal escopo foi investigar a possibilidade de ocorrência de um determinado evento. Os resultados encontrados demonstraram, a partir do efeito marginal, uma probabilidade de inserção no mercado de capitais de $6 \%$ para a amostra utilizada. Para tanto, verificou-se que o aumento de pontuações adquiridas nas questões de conhecimento financeiro refletiram em uma maior probabilidade de inserção nesse mercado. Com base nos resultados apresentados, a hipótese do estudo não é rejeitada, entretanto, destaca-se que os investimentos em educação financeira no País precisam ser aprimorados, visto que os conhecimentos financeiros dos brasileiros se apresentaram em 1,2\% abaixo da média internacional, de acordo com o relatório da Organization for Economic Cooperation and Development (2016).

Palavras-chave: Educação financeira. Capital humano. Mercado de capitais.

\section{Abstract}

Financial education and understanding of the capital markets open the doors of interaction between economic agents. The authors claim that the functioning of the capital market in Brazil is known by few, however, point out that despite these aspects, there seems to be a concern recently in attracting investors with credibility and sustainability. In this sense, the present study investigated the level of financial education of students of the business area of a public University and your relationship with the participation in the capital market. To that end, it was defined that there is no positive relationship between financial education of investors and your participation in the Brazilian capital market. For preparation of financial education, collected the data needed based on items used by Atkinson and Messy (2012), published on Organization for Economic Cooperation and Development (OECD). The method used was the multiple logistic regression, whose main scope was to investigate the possibility of occurrence of a specific event. The results demonstrated from the marginal effect insertion probability on the capital market of $6 \%$ for the sample used. For both, it was found that the increase in scores on financial knowledge acquired, reflected in a higher probability of entering this market. Based 
on the findings, the study's hypothesis is not rejected, however, that investments in financial education in the Country need to be enhanced, since the financial knowledge of Brazilians were at $1.2 \%$ below the international average, according to the report Organization for Economic Cooperation and Development (2016).

Keywords: Education. Human capital. Financial capital market.

\section{INTRODUÇÃO}

Entre os países mais populosos do mundo, o Brasil é o quinto País em maior número de habitantes, apresentando aproximadamente 206 milhões de habitantes segundo o IBGE (2016). Parte dessa população é apresentada com grande desigualdade de disponibilidade de recursos financeiros.

De acordo com os dados apresentados pela Brasil, Bolsa, Balcão - B3 (2017), fica visível que cerca de 565.263 mil brasileiros pessoas físicas participavam efetivamente do mercado de capitais. Nesse cenário, verifica-se que poucas famílias utilizam o mercado acionário como forma de investimento para preservar o consumo estável e se proteger perante as flutuações de suas rendas.

Visando à importância de se investir dentro de uma economia, Deschatre (2009) argumenta que o grau de atividade do mercado de capitais de um país está diretamente ligado ao nível de desenvolvimento de sua economia. Nesse segmento, muito se tem discutido sobre a importância da educação financeira e seus reflexos sociais e econômicos em nível nacional. A carência da saúde financeira presente na sociedade contemporânea traz como consequência a marginalização dos pequenos investidores no mercado de capitais, entretanto, a sua participação é importante para promover maior liquidez no mercado financeiro e de capitais (WISNIEWSKI, 2011).

Considerando a deficiência atual da educação financeira da sociedade, Rodrigues (2012) menciona que existem diversos pontos capazes de desestimular a participação do pequeno investidor no mercado de valores mobiliários brasileiro, como: ondas inflacionárias, estratégias políticas, crises financeiras, escândalos corporativos, supressão de garantias a investidores minoritários e crises internacionais.

Nessa perspectiva, Ramos e Moraes Junior (2012) reafirmam que o funcionamento do mercado de capitais no Brasil é conhecido por poucos, o que pode levar ao afastamento dos investidores no mercado de capitais. Contudo, Rodrigues (2012) ressalta que apesar dos aspectos citados anteriormente, parece existir uma preocupação recente em atrair esses investidores com credibilidade e sustentabilidade ao sistema financeiro nacional. 
Dessa forma, o problema no presente estudo pode ser descrito nos seguintes termos: qual a influência do nível da educação financeira dos discentes da área de negócios do Centro de Ciências Sociais Aplicadas (CCSA) da Universidade Federal da Paraíba (UFPB), Campus I sobre a probabilidade de participação no mercado de capitais?

Para responder a esse questionamento, o objetivo com a pesquisa consiste em averiguar a influência do nível de educação financeira dos discentes da área de negócios da UFPB sobre a probabilidade de participação no mercado de capitais, especificamente, com os discentes dos Cursos de Ciências Contábeis, Ciências Econômicas, Ciências Atuariais e Administração.

Buscando interpretar o ingresso dos estudantes no mercado de capitais brasileiro em ligação com o seu nível de conhecimento financeiro, estabeleceu-se a seguinte hipótese de pesquisa: a educação financeira influencia positivamente a probabilidade de participação no mercado de capitais brasileiro.

Ao fornecer evidências, por meio de modelos econométricos, de que o conhecimento sobre a educação financeira aumenta a probabilidade de participação no mercado de capitais, essa pesquisa amplia o conhecimento sobre a relação entre elementos educacionais e o mercado de capitais, expandindo, assim, a literatura nacional sobre o tema (WISNIEWSKI, 2011), que se limitou a avaliar a importância da educação financeira na gestão de finanças pessoais.

\section{FUNDAMENTAÇÃO TEÓRICA}

\subsection{EDUCAÇÃO FINANCEIRA}

O nível de educação financeira e a capacidade de fazer escolhas conscientes e seguras a respeito de finanças contribui significativamente para a solidez do mercado de capitais e para o crescimento econômico do País (WISNIEWSKI, 2011). Nesse aspecto, Rodrigues (2012) ressalta sobre a preocupação de informar e ensinar a sociedade brasileira, de forma a capacitá-los a fazerem escolhas conscientes sobre os seus investimentos, afirmando que essa atenção é incluída pelo aprimoramento do mercado de capitais. Desse modo, em decorrência do sistema econômico brasileiro estar ligado diretamente ao mercado financeiro, faz-se necessário disseminar o conhecimento sobre educação financeira para a sociedade e, dessa maneira, incentivá-la em suas escolhas e também na participação consciente nesse mercado. 
A dimensão da educação financeira é útil para os indivíduos no sentido de que é necessário elaborar planejamentos financeiros e fazer investimentos estratégicos auxiliando em suas decisões, logo, contribuem para uma adequada utilização dos recursos financeiros disponíveis aos indivíduos (GREENSPAN, 2002).

A educação financeira também é responsável por viabilizar a disseminação sobre a importância do mercado financeiro e de capitais para o crescimento social e econômico no País, tendo em vista que a liquidez do mercado é diretamente proporcional ao número de participantes ativos desse mercado. Além da liquidez, a educação financeira proporciona aos consumidores e investidores um maior controle financeiro, e, dessa maneira, torna-os capazes de gerenciar seus fluxos financeiros de modo racional e consciente durante suas tomadas de decisões na vida (MEIER; SPRENGER, 2012).

Pereira e Lucena (2014) explanaram em seu estudo que os discentes de contabilidade e engenharia com elevado nível de conhecimento financeiro possuíram uma melhor aptidão em controlar suas próprias finanças, além de compreenderem fenômenos relacionados à economia. Nesse segmento, a educação financeira contribui com o amadurecimento dos indivíduos em relação ao uso de seus recursos financeiros, auxiliando não apenas na utilização desses recursos, como também despertando o entendimento econômico e demonstrando aos consumidores como se obter rentabilidade por meio de investimentos.

Wisniewski (2011) afirma que a utilização de ferramentas essenciais da educação financeira para a gestão de finanças pessoais contribui para o hábito de poupar, além de canalizar o conhecimento de diversas modalidades de investimento, entre elas o mercado financeiro e de capitais. Segundo Kiyosaki e Lechter (2002), assuntos e ferramentas utilizados pela contabilidade e investimentos são essenciais para a gestão de finanças pessoais e empresarial, mas como esses conhecimentos financeiros são conhecidos por poucos em razão das escolas que se concentram nas habilidades acadêmicas e profissionais, deixando à mercê o desenvolvimento de habilidades financeiras.

Por esse motivo, verificou-se que a educação financeira direcionada a investimentos é um processo pelo qual consumidores aperfeiçoam seu entendimento dentro do mercado financeiro e conhecem de forma clara os produtos disponíveis, para, dessa forma, obter-se um maior crescimento quantitativo de investidores que conhecem melhor os riscos e desfrutam das oportunidades existentes tomando decisões financeiras mais assertivas e que contribuam para melhorar seu bem-estar financeiro (ORGANIZATION FOR ECONOMIC COOPERATION AND DEVELOPMENT, 2005).

Por outro lado, os consumidores, quando devidamente informados e incentivados a conhecer melhor os produtos e serviços disponíveis, tornam-se mais exigentes. 
Quanto mais os consumidores e investidores participarem e obtiverem conhecimento financeiro, maior será a demanda por produtos condizentes com as suas necessidades financeiras, assim, tornarão o mercado com maior competividade e qualidade, para que os clientes sempre tenham os produtos com características que melhor correspondam a essas demandas (BRAUNSTEIN; WELCH, 2002).

\subsection{TEORIA DO CAPITAL HUMANO}

A partir dos pesquisadores Schultz (1961) e Becker (1962), iniciou-se o argumento sobre a teoria do capital humano, que busca explicar os resultados de cada indivíduo a partir de sua escolaridade, no entanto, os que tivessem mais conhecimento e que fossem mais escolarizados eram mais propícios a elevar os ganhos de produtividade. Lucci et al. (2006) evidenciaram em seu estudo que o nível de educação financeira está ligado aos conceitos financeiros adquiridos e ao número de disciplinas relacionadas à finanças cursadas durante a graduação.

Posto isso, ao possuírem maior conhecimento e analisarem melhor o mercado, muitas vezes esses indivíduos se arriscam mais para conseguir maior rentabilidade. Van Rooij, Lusardi e Alessi (2011) afirmam que as diferentes medidas de conhecimento financeiro empregadas mostraram que a falta de alfabetização dificulta as famílias a participarem no mercado de ações.

Com base nessa afirmação, as pessoas que possuem um alto nível de educação financeira tendem a investir cada vez mais no mercado de ações, ou seja, existe forte relação entre o nível de escolaridade e o nível de educação financeira (CAMPBELL, 2006). Portanto, os investidores que possuem um nível elevado de escolaridade são mais propícios a correr riscos nesse mercado.

Por outro lado, Waltenberg (2002) faz críticas à teoria do capital humano, enfatizando que a expansão da escolaridade média não teria impreterivelmente impactos na produtividade de renda dos indivíduos. Contudo, Viana e Lima (2010) explanaram em seu estudo que, apesar das críticas e aspectos negativos demonstrados à teoria do capital humano, muito se tem enfatizado por meio do filtro de indivíduos em situações distintas, portanto, pressupõe-se que há indícios de que o capital humano, em âmbito amplo e no contexto específico na educação, é imprescindível na amplificação da produtividade econômica dos trabalhadores.

Em vista disso, apesar das críticas apresentadas, considera-se que também é relevante a utilização da teoria do capital humano para o dimensionamento da educação sob a capacidade produtiva de renda de cada indivíduo. Para tanto, Pereira e 
Lopes (2014) afirmam que a escolaridade é importante para o desenvolvimento da nação, mas antes é preciso expandir os melhores meios de educação para que os resultados futuros gerem benefícios por meio do desenvolvimento do capital humano da sociedade.

\subsection{EVIDÊNCIAS SOBRE A EDUCAÇÃO FINANCEIRA E SUA RELAÇÃO COM A PARTICIPAÇÃO DE INVESTIDORES NO MERCADO DE CAPITAIS}

Nas estimativas realizadas por Van Rooij, Lusardi e Alessi (2011), na população holandesa evidenciou-se que um maior nível de educação financeira está associado a uma maior probabilidade de investir em ações.

Em nível brasileiro, Wisniewski (2011) afirma sobre a importância de educar financeiramente os pequenos investidores, visto que foi possível verificar uma estabilidade na inserção destes na bolsa de valores, confirmando por meio de dados da participação média dos investimentos pessoas físicas na B3, entre setembro de 2010 e março de 2011, que aproximadamente 33 mil pessoas físicas deixaram a bolsa de valores.

Em estudo realizado por Xia, Wang e Li (2014) na China, foi verificado que os entrevistados que são mais confiantes com o seu nível de conhecimento financeiro são mais propensos a participarem no mercado de ações.

Alguns autores afirmam que o nível escolar elevado de um indivíduo tem conduzido a uma maior possibilidade de participação em investimentos (HALIASSOS; BERTAUT, 1995; CAMPBELL, 2006; LUSARDI; SCHERESBERG, 2013). No que se refere ao capital humano dos investidores, Cole e Shastry (2008) consideram que um ano de escolaridade de um indivíduo aumenta a sua propensão de participação do mercado financeiro de 7-8\%. Validando por meio do nível escolar, foi evidenciado que os indivíduos que dispõem formação superior possuem uma maior probabilidade em adquirir ações e empréstimos (HALIASSOS; BERTAUNT, 1995; CAMPBELL, 2006; LUSARDI; SCHERESBERG, 2013).

Os estudos produzidos na Europa afirmam que o capital humano é correlacionado com a educação financeira. Logo, essas duas variáveis dependem do mesmo conjunto para determinar a participação de ações (THOMAS; SPARATO, 2015); dessa maneira, foi possível verificar que um aumento de $1 \%$ na alfabetização financeira pode resultar em cerca de $11,3 \%$ de aumento na probabilidade de participar no mercado de ações, enquanto o capital humano mostra um efeito marginal de $1 \%$. 


\section{PROCEDIMENTOS METOdOLÓGICOS}

O universo de pesquisa em causa foram os discentes dos cursos da área de negócios (Ciências Contábeis, Ciências Atuariais, Ciências Econômicas e Administração) do CCSA da UFPB Campus I, constituindo-se o procedimento de coleta de dados de forma presencial ao máximo de respondentes possível.

\subsection{PROCEDIMENTO DE COLETA DE DADOS}

Para a progressão da pesquisa, foram aplicados 302 questionários seguindo modelo de aplicação survey aos respondentes. Em razão da especificidade da pesquisa, foi elaborado um modelo de questionário para a obtenção desses dados, como informações demográficas, financeiras e do nível de conhecimento financeiro da amostra selecionada.

O questionário teve como primeiro objetivo traçar o perfil dos respondentes, recolhendo informações como a escolaridade, o posicionamento no mercado de capitais e a quantidade de disciplinas cursadas relacionadas à área financeira, além de detectar outras características individuais (sexo, idade, estado civil, valor poupado e quantidade de dependentes financeiros) de cada pesquisado, conforme se demonstra no Quadro 1:

Quadro 1 - Perfil do respondente

\begin{tabular}{|c|c|c|}
\hline Quesitos & $\mathrm{O}$ que mede & Escala \\
\hline 1 & Qual o sexo do respondente & $\begin{array}{l}\text { Variável dicotômica simples, qualitativa, } \\
\text { escala nominal }\end{array}$ \\
\hline 2 & A idade do respondente & Escala de razão \\
\hline 3 & O estado civil do respondente & $\begin{array}{l}\text { Variável dicotômica simples, qualitativa, } \\
\text { escala nominal }\end{array}$ \\
\hline 4 & Possui dependente financeiro & Escala de razão \\
\hline 5 & A renda do respondente & Escala de razão \\
\hline 6 & $\begin{array}{l}\text { O valor poupado da renda mensal líquida } \\
\text { do respondente }\end{array}$ & Escala de razão \\
\hline 7 & A escolaridade do respondente & Variável categórica nominal \\
\hline 8 & O curso que o respondente está cursando & $\begin{array}{l}\text { Escala de múltipla escolha com resposta } \\
\text { única (escolha múltipla simples) }\end{array}$ \\
\hline 9 & Qual o semestre que está cursando & Escala de razão \\
\hline 10 & Quais disciplinas & $\begin{array}{l}\text { Escala de múltipla escolha com várias res- } \\
\text { postas (escolha múltipla simples) }\end{array}$ \\
\hline 17 & $\begin{array}{l}\text { Participação no mercado de capitais do } \\
\text { respondente }\end{array}$ & $\begin{array}{l}\text { Escala de múltipla escolha com resposta } \\
\text { única (escolha múltipla simples) }\end{array}$ \\
\hline 18 & Receber resultado por e-mail & $\begin{array}{l}\text { Escala de múltipla escolha com resposta } \\
\text { única (escolha múltipla simples) }\end{array}$ \\
\hline
\end{tabular}

Fonte: os autores. 
Além traçar o perfil dos respondentes, foram coletadas informações para a criação do nível de conhecimento financeiro. Dessa forma, para se identificar o nível de conhecimento financeiro, foi elaborado um Indicador de Educação Financeira (IEF), que representou o nível de educação financeira de cada respondente, por meio de seis quesitos (com escore de 0 a 6 ), com base na quantidade de acertos ( 1 acerto = 1 escore do IEF) das questões descritas no Quadro 2:

Quadro 2 - Conhecimentos mensurados para o IEF e suas respectivas escalas

\begin{tabular}{|c|l|l|}
\hline Quesitos & \multicolumn{1}{|c|}{ O que mede } & \multicolumn{1}{|c|}{ Escala } \\
\hline 1 & $\begin{array}{l}\text { Conhecimento sobre diferenças no risco entre } \\
\text { renda fixa e variável }\end{array}$ & $\begin{array}{l}\text { Escala de múltipla escolha com respos- } \\
\text { ta única }\end{array}$ \\
\hline 2 & $\begin{array}{l}\text { Conhecimento financeiro, juros compostos e } \\
\text { sistemas de amortização }\end{array}$ & $\begin{array}{l}\text { Escala de múltipla escolha com respos- } \\
\text { ta única }\end{array}$ \\
\hline 2 & Conhecimento do risco de ações & Escala de verdadeiro ou falso \\
\hline 4 & $\begin{array}{l}\text { Conhecimentos sobre o valor do dinheiro no } \\
\text { tempo, inflação e matemática financeira }\end{array}$ & $\begin{array}{l}\text { Escala de múltipla escolha com respos- } \\
\text { ta única }\end{array}$ \\
\hline 5 & $\begin{array}{l}\text { Conhecimentos sobre alternativas de investimen- } \\
\text { tos em relação ao custo de oportunidade }\end{array}$ & $\begin{array}{l}\text { Escala de múltipla escolha com respos- } \\
\text { ta única }\end{array}$ \\
\hline 6 & $\begin{array}{l}\text { Conhecimento sobre o Modelo de Precificação } \\
\text { de Ativos Financeiros (CAPM) }\end{array}$ & $\begin{array}{l}\text { Escala de múltipla escolha com respos- } \\
\text { ta única }\end{array}$ \\
\hline
\end{tabular}

Fonte: os autores.

Os quesitos utilizados para a elaboração do IEF foram criados com base no estudo piloto realizado por Atkinson e Messy (2012), que teve por objetivo analisar variações no conhecimento financeiro, no comportamento e na atitude entre 14 países, publicado na OECD Working Papers on Finance, Insurance and Private Pensions.

Para a mensuração do nível de educação financeira, foram abordados conceitos fundamentados em questões de conhecimentos em: matemática básica (division), valor do dinheiro no tempo (time-value of Money), matemática financeira (interest paid on a loan, calculation of interest plus principle, compound interest), risco e retorno (risk and return), inflação (definition of inflation) e ferramentas para a otimização de carteiras (diversification).

Os conceitos apresentados no Quadro 2 foram mensurados a partir de cinco questões objetivas com escala de múltipla escolha (com resposta única) e uma com escala de verdadeiro ou falso, atribuindo resposta única e com mesmo peso. 


\subsection{TESTE DE HIPÓTESES}

O teste de hipótese foi realizado por meio do método de regressão logística múltipla (modelo logit), que segundo Hair Junior et al. (1998), tem a intenção de investigar a possibilidade de ocorrência de um determinado evento em estudo.

Bender Filho, Bagolin e Comim (2010) explanaram que a maneira mais adequada para ajustar um modelo com variável dependente binária é utilizando os modelos probit ou logit. Logo, a análise econométrica foi desenvolvida pela equação de estimação a partir de uma regressão logit, sendo o modelo mais utilizado para explorar a relação entre uma ou mais variáveis explicativas e uma variável dependente dummy.

A variável dependente é a participação no mercado de capitais, cuja natureza é binária (valores 0 ou 1). Thomas e Sparato (2015) evidenciaram que a inclusão de variáveis independentes idade, estado civil, sexo, capital humano e educação financeira proporcionou resultados mais robustos no modelo binário utilizado.

O modelo empírico da pesquisa é apresentado por meio da Equação 1:

$$
\begin{gathered}
P_{i}=\frac{1}{1+e^{-\left(\beta_{0}+\beta_{1} I E F_{i}+\beta_{2} C H_{i}+\beta_{8} D C F_{i}+\beta_{4} V P_{i}+\beta_{5} H O_{i}+\beta_{6} I D_{i}+\beta_{7} E C_{i}+\beta_{8} D F_{i}+\varepsilon_{i}\right)}} \\
\begin{aligned}
L i=\ln \frac{p}{1-p}= & \beta 1+\beta 2 I E F i+\beta 3 C H i+\beta 4 D C F i+\beta 5 V P i+\beta 6 H O i+\beta 7 I D i \\
& +\beta 8 E C i+B 9 D F i+u i(1)
\end{aligned}
\end{gathered}
$$

Por meio da Equação 1, pode-se identificar como foi realizada a categorização das variáveis utilizadas no estudo: $\mathrm{P}_{\mathrm{i}}$ indica a probabilidade de o indivíduo participar do mercado de capitais (Equação 1); IEF (Indicador de Educação Financeira) - equivale à variável independente principal do modelo, que representa o nível de educação financeira de cada discente (com escore de 0 a 6); e CH (Capital Humano) reflete o nível de escolaridade do último ano de estudo cursado (por exemplo, o nível de ensino médio completo corresponde aproximadamente a 11 anos de estudo), portanto, se o respondente possui nível superior incompleto e está no segundo semestre (primeiro ano cursado), possui o equivalente a 12 anos de escolaridade.

As demais variáveis categorizadas são: Disciplinas Correlatas à Finanças (DCF) - representam a quantidade de disciplinas ligadas a finanças cursadas pelos discentes durante a graduação; Valor Poupado (VP) - demonstra o valor poupado da renda líquida mensal em reais (por exemplo, a multiplicação do percentual poupado 
escolhido de $10 \%$ ou $0 \%$ vezes a renda mensal líquida do respondente de $\mathrm{R} \$ 1.000,00$ reais equivale a um valor poupado de $\mathrm{R} \$ 100,00$ reais ou $\mathrm{R} \$ 0,00$ reais; Homem (HO) - representa a variável binária (dummy) do estatuto do sexo masculino (se for homem atribui-se o valor 1, caso contrário, o valor 0); Idade (ID) - a variável explicativa representativa da idade dos indivíduos; Estado Civil (EC) - a variável independente binária (dummy) representada pelo estado civil do indivíduo (se for casado será classificado com o valor 1, caso contrário, com o valor 0); Dependentes Financeiros (DF) - a variável explicativa que representa o número de dependentes financeiros; $\varepsilon$ - o termo de erro estocástico da regressão; e $\beta$ - o coeficiente das variáveis independentes.

Dessa forma, a significância do estimador definirá a rejeição da hipótese do estudo, ou seja, se a participação no mercado de capitais dos discentes da área de negócios da UFPB dimensiona efeitos de seus conhecimentos sobre educação financeira adquirida ao longo de sua vida e formação acadêmica.

\section{ANÁLISE DOS RESULTADOS}

\subsection{ANÁLISE DESCRITIVA DOS DADOS}

Por meio das 302 observações coletadas e apresentadas na Tabela 1, observou-se que 275 pesquisados não participam no mercado de capitais, ou seja, cerca de 91\% da amostra apreciada. Por conseguinte, participam desse mercado apenas 27 indivíduos, representando 9\% do total da amostra. Deschatre (2009) sustenta que mesmo contando com os avanços tecnológicos de informação, a ausência de informação aparenta ser a causadora do pouco número de investidores no mercado acionário brasileiro.

Tabela 1 - Participação da amostra no mercado de capitais

\begin{tabular}{lrrrrrr}
\hline Posicionamento & & Homem & & Mulher & \multicolumn{2}{r}{ Total geral } \\
\hline Participa & 17 & $6 \%$ & 10 & $3 \%$ & 27 & $9 \%$ \\
\hline Não participa & 148 & $49 \%$ & 127 & $42 \%$ & 275 & $91 \%$ \\
\hline Total geral & 165 & $55 \%$ & 137 & $45 \%$ & 302 & $100 \%$ \\
\hline Fonte: os autores. & & & & & &
\end{tabular}

Segundo dados apresentados pela Brasil, Bolsa, Balcão - B3 (2017), na Tabela 2 o Estado da Paraíba, no qual foi realizada a coleta de dados, possui cerca de 2.707 investidores pessoa física no mercado acionário; logo, os 27 participantes da 
amostra específica equivalem a 1\% do total de participantes desse mercado no Estado da Paraíba.

É relevante frisar que ao examinarmos os gêneros dos respondentes apresentados na Tabela 1 , percebeu-se que os 27 participantes do mercado de capitais, em sua maioria, são compostos por homens, tendo em vista que eles representam 62,96\%, enquanto as mulheres apenas 37,04\%.

De acordo com a Tabela 2, o perfil por sexo em nível paraibano está em 79,61\% para os homens e 20,39\% para as mulheres. Observa-se, também, que as estatísticas apresentadas demonstram que o perfil do investidor em nível estadual e nacional está aproximado, retratando mais uma vez a realidade da amostra com os cenários estadual e nacional.

Tabela 2 - Perfil do investidor em níveis estadual e nacional

\begin{tabular}{lrrrr}
\hline Perfil PF por Sexo & Paraíba & \multicolumn{2}{c}{ Brasil } \\
& Investidores & \% & Investidores & \% \\
\hline Homens & 2.155 & $79,61 \%$ & 435.107 & $76,97 \%$ \\
\hline Mulheres & 552 & $20,39 \%$ & 130.157 & $23,03 \%$ \\
\hline Total & 2.707 & $100 \%$ & 565.263 & $100 \%$ \\
\hline
\end{tabular}

Fonte: adaptada da Brasil, Bolsa, Balcão - B3 (2017).

Para a consecução de uma melhor visualização das variáveis coletadas, evidenciou-se, na Tabela 3, uma estatística descritiva dos dados utilizados neste estudo por meio da média, desvio padrão, valor mínimo e valor máximo de cada variável:

Tabela 3 - Estatística descritiva da amostra

\begin{tabular}{lrrrr}
\hline Variáveis & Média & Desvio padrão & Min & Max \\
\hline Indicador de Educação Financeira (IEF) & 3,84 & 1,19 & 0 & 6 \\
Capital Humano (CH) & 14,28 & 1,28 & 12 & 18 \\
Disciplinas Correlatas a Finanças (DCF) & 2,32 & 1,36 & 0 & 6 \\
Valor Poupado (VP) & 233,98 & 781,12 & 0 & 8.400 \\
Homem (HO) & 0,55 & 0,50 & 0 & 1 \\
Idade (ID) & 24,96 & 5,80 & 17 & 52 \\
Estado Civil (EC) & 0,22 & 0,41 & 0 & 1 \\
Dependentes Financeiros (DF) & 0,26 & 0,62 & 0 & 4 \\
Participação no Mercado de Capitais (Y) & 0,09 & 0,29 & 0 & 1 \\
\hline Fonte: os autores. & & &
\end{tabular}


Com base na Tabela 3, evidenciou-se que o nível de conhecimento financeiro dos discentes correspondeu em média a um escore de 3,84, convertendo-se em um aproveitamento de 64\% em relação às questões utilizadas no questionário para a estimação do IEF. No que diz respeito ao capital humano, constatou-se que a amostra possui aproximadamente uma média de escolaridade de 14 anos, portanto, os discentes em média estão cursando o terceiro ano do ensino superior, apresentando um desvio padrão de apenas um ano. No que se refere às disciplinas correlatas a finanças evidenciadas no questionário, os alunos em média cursaram cerca de duas disciplinas, contendo por volta de uma disciplina de desvio padrão.

Em relação às médias das características dos indivíduos do presente estudo, foi identificado que $55 \%$ da amostra correspondem a indivíduos do sexo masculino. Constatou-se que, em média, 22\% são casados ou possuem uma união estável. Pode-se observar que os pesquisados alcançaram uma idade média de 25 anos, além de um valor médio e máximo poupado no que se refere às suas rendas mensais líquidas no valor de R \$ 233,98 reais e R \$ 8.400,00 reais, respectivamente. Além disso, é notável que em média $26 \%$ dos pesquisados possuem um dependente financeiro.

As distribuições apresentadas pela estatística descritiva fornecem uma visão interessante da amostra total estudada, mas no que diz respeito ao Gráfico 1, pode-se focalizar a proporção de que existem diferenças notáveis entre os sexos a respeito do nível do conhecimento financeiro para a amostra do Estado da Paraíba e do estudo realizado pela Organization for Economic Cooperation and Development (2016) com respondentes de 30 países, incluindo o Brasil:

Gráfico 1 - Pontuação média (\%) do conhecimento financeiro por gênero

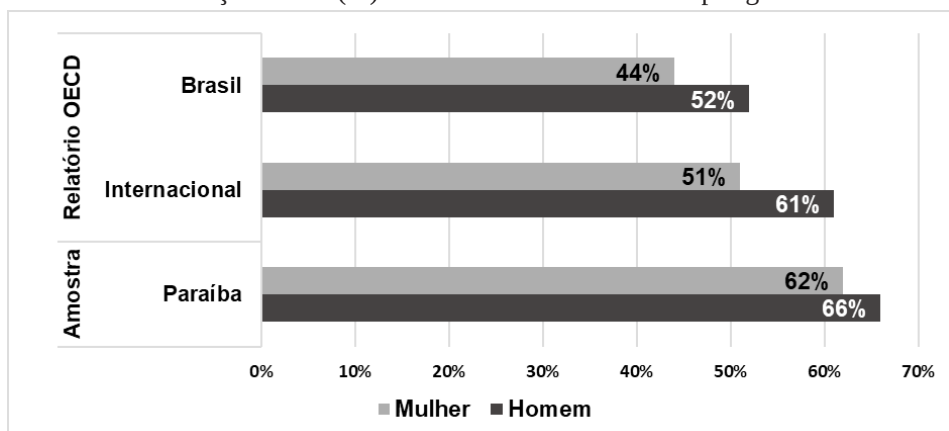

Fonte: adaptado da Organization for Economic Cooperation and Development (2016).

Os achados sobre o nível de conhecimento financeiro na amostra do Estado da Paraíba expressaram uma diferença de 4\% entre os gêneros; já no relatório da Or- 
ganization for Economic Cooperation and Development (2016) demonstraram uma diferença de $8 \%$ para o Brasil e de 10\% em nível internacional. Logo, pode-se afirmar que existem diferenças expressivas entre a proporção de homens e mulheres relativas às pontuações adquiridas nas questões de conhecimento financeiro, tanto em nível nacional quanto em nível internacional.

Nessa perspectiva, Atkinson e Messy (2012) mostraram-se preocupados e concluíram que alguns países precisam se empenhar para garantir que as mulheres não fiquem para trás, pois elas obtiveram, na maioria dos países estudados, níveis mais baixos de conhecimento financeiro em relação aos homens.

\subsection{ANÁLISE DO MODELO EMPÍRICO}

Utilizou-se, neste estudo, o método de regressão logística múltipla, cujo principal escopo foi a realização de cálculos probabilísticos, no caso específico, a probabilidade dos 302 discentes participarem do mercado de capitais, dado o seu conhecimento sobre educação financeira e suas características individuais.

Conforme Freitas (2013), as funções de ligação da regressão logit possuem como variável dependente (dicotômica) uma variável binária. A variável dummy representa o posicionamento de participação no mercado de capitais (Participa=1; Não participa=0). A função linear dos regressores é usada pelo logit, dessa maneira, para o prosseguimento do cálculo de probabilidade, enfatiza-se que a função de probabilidade subjacente ao modelo logit é dada pela distribuição logística (GUJARATI; PORTER, 2011).

Nesse contexto, Mesquita (2014) evidencia que os coeficientes considerados no modelo de regressão logit indicam uma variabilidade no que diz respeito à probabilidade de ocorrência de um determinado evento, em consonância da variação de uma unidade na variável independente.

Para estimar o modelo logit apresentado na Tabela 3, foram necessárias seis interações. A função do teste $L R$ Chi2 (qui-quadrado) é aproximadamente a mesma utilizada no teste $F$ convencional, resultando em um valor igual a 31,40. Esse valor indica que os coeficientes são significativos para explicar a probabilidade dos indivíduos estarem no mercado de capitais. Por meio do teste da razão de verossimilhanças, verifica-se a hipótese de nulidade de que todos os coeficientes, nesse sentido, o valor 0,0001 da estatística Prob $\left(X^{2}\right)$, indica que se pode rejeitar a $1 \%$ a hipótese de que todos os coeficientes sejam iguais a zero. 
Sob outra perspectiva, a Tabela 4 evidencia que o IEF foi significante, logo, por meio do cálculo do antilogaritmo $\left(e^{(0,8786081)}=2,41\right)$, verifica-se que se o pesquisado obtém 1 escore de conhecimento sobre educação financeira, as chances da participação no mercado de capitais praticamente dobram, sendo mantidos os demais fatores constantes. Nesse sentido, a Tabela 4 reflete que a maioria dos estimadores do modelo possuem relação positiva com variável explicada existente, exceto as variáveis DCF e ID:

Tabela 4 - Modelo Logit

\begin{tabular}{lrrrr}
\hline Participação no mercado de capitais & Estimador & Erro & z-teste & Prob. \\
\hline IEF & 0,8786081 & 0,2497662 & 3,52 & 0,000 \\
CH & 0,4788647 & 0,2153994 & 2,22 & 0,026 \\
DCF & $-0,4807266$ & 0,2116324 & $-2,27$ & 0,023 \\
VP & 0,0003959 & 0,000217 & 1,82 & 0,068 \\
HO & 0,0979083 & 0,4677517 & 0,21 & 0,834 \\
ID & $-0,0141759$ & 0,055311 & $-0,26$ & 0,798 \\
EC & 0,0424349 & 0,6409157 & 0,07 & 0,947 \\
DF & 0,3193927 & 0,413686 & 0,77 & 0,440 \\
Constante & $-11,82164$ & 3,045841 & $-3,88$ & 0,000 \\
\hline Fonte: os autores. & & & &
\end{tabular}

N. de observações $=302$.

$\chi^{2}=31.40$ (Lr chi2), Prob $\left(\chi^{2}\right)=0,0001$, Pseudo R2 = 0,1726.

Logo após a estimação do modelo logit, utilizaram-se outras medidas para verificar a qualidade de ajustamento do modelo. Por intermédio da Tabela 5, verificou-se a qualidade do ajustamento do modelo a partir de uma matriz de contingência, especificando o valor e a determinação de uma variável para obter um resultado previsto positivo. Para tal fim, efetuou-se a classificação de cada observação como positiva, se a sua possibilidade prevista for maior que 0,5 (padrão), comparando-se posteriormente com os valores reais observados:

Tabela 5 - Matriz de contingência

\begin{tabular}{rrrrr}
\hline \multicolumn{1}{c}{ Classificação } & Participa (D) & & Não participa ( D) & \multicolumn{2}{c}{ Total } \\
\hline$(+)$ & & 4 & 1 & 5 \\
$(-)$ & 23 & 274 & 297 \\
& & 27 & 275 & 302 \\
\hline
\end{tabular}

Fonte: os autores.

Nota: Classificação Correta = 92,05\% ((4+274)/302). 
Além do teste padrão com referência de 0,5, realizaram-se os mesmos cálculos por meio do teste de sensibilidade com o ponto de corte de 0,91, com base no nível de acerto, representando de fato a predominância da resposta sobre a outra, obtendo-se uma classificação correta de 91,06\%, verificando uma melhor acurácia no modelo.

Dentro dessa mesma perspectiva, conforme a Tabela 6, são apresentados os diversos cálculos probabilísticos em razão da Tabela 5 da matriz de contingência. No que se refere aos valores corretamente classificados, de modo geral, o modelo prevê 92,05\% das observações corretamente no teste padrão, e 91,06\% no teste de sensibilidade de predominância, ou seja, dos não participantes.

Tabela 6 - Probabilidade de eventos

\begin{tabular}{lrr}
\hline \multicolumn{1}{c}{ Descrição } & Argumento & Prob.(\%) \\
\hline Sensibilidade & $\operatorname{Pr}(+\mid \mathrm{D})$ & $14,81 \%$ \\
Especificidade & $\operatorname{Pr}(-\mid \sim \mathrm{D})$ & $99,64 \%$ \\
O valor preditivo positivo & $\operatorname{Pr}(\mathrm{D} \mid+)$ & $80 \%$ \\
O valor preditivo negativo & $\operatorname{Pr}(\sim \mathrm{D} \mid-)$ & $92,26 \%$ \\
Falso (+) para ( D) verdadeiro & $\operatorname{Pr}(+\mid \sim \mathrm{D})$ & $0,36 \%$ \\
Falso (-) para (D) verdadeiro & $\operatorname{Pr}(-\mid \mathrm{D})$ & $85,19 \%$ \\
Falso (+) classificado (+) & $\operatorname{Pr}(\sim \mathrm{D} \mid+)$ & $20 \%$ \\
Falso (-) classificado (-) & $\operatorname{Pr}(\mathrm{D} \mid-)$ & $7,74 \%$ \\
Classificação correta & & $92,05 \%$ \\
\hline Fonte: os autores. & & \\
Nota: Classificação (+) se a predição de $\operatorname{Pr}(\mathrm{D})>=0,5$ & & \\
Verdadeiro “D” definido como Participação no Mercado de $\operatorname{Capitais}=0$. &
\end{tabular}

No que diz respeito ao modelo empírico substancializado na regressão logit, é indiscutível que o entendimento e a interpretação dos coeficientes derivam das estimativas em relação aos sinais positivo e negativo, destacando a probabilidade positiva ou negativa de verificação do acontecimento do evento, ou seja, a participação no mercado de capitais. Dessa maneira, verificou-se o efeito marginal do modelo logit na Tabela 6, retratando novamente que a maioria dos coeficientes possui sinais positivos, apresentando relações positivas com a variável explicada. 
Tabela 7 - Efeito Marginal do modelo logit

\begin{tabular}{crrrrr}
\hline $\begin{array}{c}\text { Participação no Mercado de } \\
\text { Capitais }\end{array}$ & $\mathbf{d y} / \mathbf{d x}$ & Erro & $\mathbf{z}$ & $\mathbf{P}>\mathbf{z}$ & $\mathbf{X}$ \\
\hline IEF & 0,0461027 & 0,01109 & 4,16 & 0,000 & 3,84437 \\
CH & 0,0251272 & 0,01155 & 2,17 & 0,030 & 14,2815 \\
DCF & $-0,0252249$ & 0,01138 & $-2,22$ & 0,027 & 2,31788 \\
VP & 0,0000208 & 0,00001 & 1,71 & 0,088 & 234 \\
HO & 0,0051182 & 0,02434 & 0,21 & 0,833 & 0,546358 \\
ID & $-0,0007438$ & 0,00289 & $-0,26$ & 0,797 & 24,9603 \\
EC & 0,0022505 & 0,03432 & 0,07 & 0,948 & 0,218543 \\
DF & 0,0167593 & 0,02167 & 0,77 & 0,439 & 0,264901 \\
\hline
\end{tabular}

Fonte: os autores.

Nota: $y=\operatorname{Pr}($ Participação no Mercado de Capitais) $($ preditivo $)=0,05555926$.

No entanto, examinou-se a magnitude e relevância da educação financeira retratada na Tabela 7 por meio do efeito marginal do presente modelo. No efeito marginal das variáveis do modelo, a variação no logit é medida pelos coeficientes, estimando uma variação unitária da variável explicada. Assim, se o discente participa no mercado de capitais, ocorre o aumento do logit. Nesse sentido, a probabilidade de um indivíduo mediano em participar no mercado de capitais é de aproximadamente 6\% para a amostra específica.

Ao analisar as variáveis representativas destacadas na Tabela 7, considerando que o dy/dx representa a variação discreta da variável dummy de 0 para 1, notou-se que 1 escore do IEF aumenta a probabilidade de o estudante participar no mercado de capitais em cerca de 4,61\%. Assim, evidenciou-se que não se rejeita a hipótese de que o Indicador de Educação Financeira (IEF) tem uma relação positiva com a participação no mercado de capitais dos discentes da área de negócios da UFPB.

Em relação ao capital humano, um indivíduo que tem um ano a mais de escolaridade, tem uma probabilidade de participação de 2,51 pontos percentuais. Além disso, observa-se que cada valor unitário das variáveis valor poupado, homem, estado civil e dependentes financeiros contribui respectivamente em 0,002\%, 0,512\%, 0,225\% e 1,676\% na participação dos discentes nesse mercado. É notório que o número de disciplinas correlatas a finanças e à idade dos respondentes possui relação negativa no aumento da probabilidade de inserção no mercado de capitais. Fica evidente que esses valores são tomados isoladamente, ressaltando, por oportuno, que se o investidor possuir poucos conhecimentos em educação financeira, terá uma propensão menor de participação nesse mercado. 
O cálculo da estatística Count R2 indicou que o modelo acertou 92,1\% das previsões realizadas, sendo, assim, representada por meio da razão do número de previsões corretas e do número de observações do modelo. Desse modo, pode-se verificar de forma simples a robustez e o nível de ajustamento do modelo.

Para verificar a amostra total de forma mais complexa e sofisticada do ponto de vista estatístico, utilizou-se o teste de Hosmer-Lemeshow para observar o nível de adequação do modelo aos dados. A partir do teste, as observações foram divididas em 10 classes. Para cada classe, foi realizada uma estimativa por meio da distribuição qui-quadrado dos valores estimados pelo modelo e dos valores reais coletados, e, por fim, as comparações.

O output encontrado do teste de Hosmer-Lemeshow foi de 0,3073, considerando que o $p$-valor foi maior que 0,05 ; verifica-se que não existe diferença entre os valores estimados e os valores observados. Portanto, não se rejeita a hipótese nula de forma que existe diferença entre os valores reais e os valores estimados pelo modelo, comprovando um bom nível de acurácia e precisão na estimativa de probabilidade.

Tabela 8 - Probabilidade de participação dos indivíduos com escore igual a 6 do IEF

\begin{tabular}{crrrrr}
\hline $\begin{array}{c}\text { Participação no mercado de } \\
\text { capitais }\end{array}$ & $\mathbf{d y} / \mathbf{d x}$ & Erro & $\mathbf{z}$ & $\mathbf{P}>\mathbf{z}$ & $\mathbf{X}$ \\
\hline IEF & 0,1775378 & 0,08041 & 2,21 & 0,027 & 6 \\
CH & 0,0967628 & 0,0463 & 2,09 & 0,037 & 14,2815 \\
DCF & $-0,097139$ & 0,04623 & $-2,10$ & 0,036 & 2,31788 \\
VP & 0,00008 & 0,00004 & 1,83 & 0,068 & 234 \\
HO & 0,019743 & 0,09372 & 0,21 & 0,833 & 0,546358 \\
ID & $-0,0028645$ & 0,01119 & $-0,26$ & 0,798 & 24,9603 \\
EC & 0,0086193 & 0,13087 & 0,07 & 0,947 & 0,218543 \\
DF & 0,0645387 & 0,08551 & 0,75 & 0,450 & 0,264901 \\
\hline Fonte: 0 autores. & & & & &
\end{tabular}

Fonte: os autores.

Nota: $y=\operatorname{Pr}$ (Participação no Mercado de Capitais) (preditivo) = 0,28106409.

No entanto, após os testes de robustez, analisou-se na Tabela 8 que se um discente obtiver um resultado máximo (escore=6) no Indicador de Educação Financeira (IEF), mantendo o mesmo ponto médio nas demais variáveis independentes, ele terá uma probabilidade de $28,11 \%$ de ser inserido no mercado de capitais. 
Tabela 9 - Probabilidade de participação dos indivíduos com score igual a 0 do IEF

\begin{tabular}{crrrrr}
\hline $\begin{array}{c}\text { Participação no mercado de } \\
\text { capitais }\end{array}$ & $\mathbf{d y} / \mathbf{d x}$ & Erro & $\mathbf{z}$ & $\mathbf{P}>\mathbf{z}$ & $\mathbf{X}$ \\
\hline IEF & 0,0017568 & 0,00155 & 1,13 & 0,257 & 0 \\
CH & 0,0009575 & 0,00113 & 0,85 & 0,397 & 14,2815 \\
DCF & $-0,0009612$ & 0,00112 & $-0,86$ & 0,392 & 2,31788 \\
VP & $7.92 \mathrm{e}-07$ & 0,00000 & 0,76 & 0,445 & 234 \\
HO & 0,000195 & 0,00097 & 0,20 & 0,840 & 0,546358 \\
ID & $-0,0000283$ & 0,00011 & $-0,25$ & 0,802 & 24,9603 \\
EC & 0,0000859 & 0,00131 & 0,07 & 0,948 & 0,218543 \\
DF & 0,0006386 & 0,00103 & 0,62 & 0,536 & 0,264901 \\
\hline
\end{tabular}

Fonte: os autores.

Nota: $y=\operatorname{Pr}($ Participação no Mercado de Capitais) $($ preditivo $)=0,00200353$.

De outro modo, verificou-se na Tabela 9 que se o respondente obtiver um resultado mínimo (escore $=0$ ) no Indicador de Educação Financeira (IEF), preservando os mesmos valores do ponto médio das demais variáveis explicativas, ele terá uma probabilidade de ser inserido no mercado de capitais de apenas $0,2 \%$.

\section{CONCLUSÃO}

A pesquisa buscou, essencialmente, identificar quais as magnitudes da propensão de um indivíduo participar no mercado de capitais, dado os seus conhecimentos financeiros, nível de escolaridade, a estabilidade financeira e suas características individuais coletadas, com a finalidade de identificar as necessidades e lacunas no fornecimento de educação financeira e contribuir no desenvolvimento de políticas ou estratégias nacionais para a inserção de novos investidores, de acordo com o desenvolvimento de recomendações e princípios da OECD (ATKINSON; MESSY, 2012).

Os cálculos probabilísticos do efeito marginal demonstraram que a probabilidade de participação no mercado de capitais dos discentes no ponto médio foi de 6\% para a amostra, revelando aumentos positivos significativos consoantes com a literatura internacional (HALIASSOS ; BERTAUT, 1995; CAMPBELL, 2006; VAN ROOIJ; LUSARDI; ALESSI, 2011; LUSARDI; SCHERESBERG, 2013; XIA; WANG; LI, 2014), a título de exemplo, no estudo realizado na Europa que resultou em uma propensão de 11,3\% de um indivíduo ser inserido no mercado de ações (THOMAS; SPARATO, 2015). 
Evidenciando a robustez do modelo a partir dos testes, identificou-se que se um indivíduo com valor poupado de sua renda mensal líquida obtiver um conhecimento financeiro máximo no IEF (escore=6), mantendo os demais fatores constantes, obterá uma probabilidade de 28,11\% de ser inserido no mercado de capitais.

Com base nas análises e resultados apresentados, a hipótese do estudo não é rejeitada, validando de que existe relação positiva entre o nível de educação financeira dos discentes da área de negócios da UFPB e a participação no mercado de capitais brasileiro. Apesar da relação positiva, destaca-se que os investimentos em educação financeira no País precisam ser aperfeiçoados, uma vez que os conhecimentos financeiros dos brasileiros apresentados no relatório da Organization for Economic Cooperation and Development (2016) ficaram em 1,2\% abaixo da média internacional.

Outro ponto de vista a ser considerado é a diferença da proporcionalidade de conhecimento financeiro entre os homens e as mulheres. No Brasil e em outros países (HALIASSOS; BERTAUT, 1995; FONSECA et al., 2012) as mulheres obtiveram baixos níveis de conhecimento financeiro em relação aos homens (ATKINSON; MESSY, 2012; OECD, 2016). Em vista disso, fica evidente que o Brasil precisa progredir também nesses aspectos para garantir uma estabilidade financeira nacional sem desigualdade de gêneros.

Destaca-se como limitação a dificuldade de acesso aos dados de investidores ativos no mercado de capitais brasileiro de forma aleatória; em face à mesma realidade em análises realizadas por Thomas e Sparato (2015), o estudo limitou-se aos discentes da área de negócios da UFPB. As conclusões elaboradas ficam restritas para a amostra investigada, assim, no que se refere à existência de poucos discentes nesse mercado, considera-se que as limitações não invalidam os resultados encontrados, logo, os achados são relevantes para o desenvolvimento e a disseminação do conhecimento na sociedade.

Observando a importância da abordagem do assunto educação financeira e mercado de capitais no País, é imprescindível investigar e apresentar estudos sobre o presente tema, visto que a existência de estudos nacionais sobre a inserção da população em investimentos trata diversas contribuições para a sustentabilidade do sistema financeiro e para o desenvolvimento econômico do País. Os resultados encontrados permitem que a comparação entre países e estados seja utilizada para desenvolver soluções para o aumento da educação financeira e a estabilidade financeira das populações investigadas (ORGANIZATION FOR ECONOMIC COOPERATION AND DEVELOPMENT, 2016). 
Para a realização de estudos futuros se recomendam a ampliação da amostra e a inclusão de novas variáveis determinantes para a inserção de indivíduos no mercado financeiro e de capitais, além da utilização e comparação com outros estudos e modelos econométricos.

\section{REFERÊNCIAS}

ATKINSON, A.; MESSY, F. Measuring Financial Literacy: Results of the OECD INFE Pilot Study. OECD Working Papers on Finance, Insurance and Private Pensions, OECD: Publishing, Paris, n. 15, 2012.

BECKER, G. Investiment in Human Capital: A Theoretical Analysis. The Journal of Political Economy, v. 70, i. 5, p. 9-49, 1962.

BENDER FILHO, R.; BAGOLIN, I, P.; COMIM, F. V. Determinantes da permanência na condição de pobreza crônica: aplicação do modelo logit multinomial. Texto para discussão, Porto Alegre: PUC-RS, n. 7, 2010.

BRASIL, BOLSA, BALCÃO - B3. Histórico pessoa física. 2017. BRAUNSTEIN, S.; WELCH, C. Financial literacy: An overview od practice, research, and policy. Federal Reserve Bulletin, Estados Unidos, p. 445-457, 2002.

CAMPBELL, J. Y. Household Finance. The Journal of Finance, v. 61, i. 4, p. 1553-1604, 2006.

COLE, S.; SHASTRY, G. K. If you are so smart, why aren't you rich? The effects of education, financial literacy and cognitive ability on financial market participation. Working Paper, Harvard Business School, Wellesley College (unpublished), p. 9-71, 2008.

DESCHATRE, G. A. Investimento em ações: para os momentos de crise e de crescimento. Rio de Janeiro: Thomas Nelson Brasil, 2009.

FONSECA, R. et al. What explains the gender gap in financial literacy? The role of household decision making. Journal of Consumer Affairs, v. 46, i. 1, p. 90-106, 2012.

FREITAS, L. dos R. Comparação das Funções de Ligação Logit e Probit em Regressão Binária Considerando Diferentes Tamanhos Amostrais. 2013. Tese (Doutorado)-Universidade Federal de Viçosa, 2013. 
GREENSPAN, A. Financial Literacy: A Tool for Economic Progress. The Futurist, v. 36, i. 4, p. 20237-20241, 2002.

GUJARATI, D. N.; PORTER, D. C. Econometria básica. 5. ed. Porto Alegre: AMGH, 2011.

HAIR JUNIOR, J. F. et al. Multivariate analyses data. $5^{a}$ Ed. New Jersey: Princeton University Press, 1998.

HALIASSOS, M.; BERTAUT, C. Why do so few hold stocks? The Economic Journal, v. 105, i. 432, p. 1110-1129, 1995.

IBGE. Estimativas da população residente no Brasil e Unidades da Federação com data de referência em $\mathbf{1}^{\mathbf{0}}$ de julho de 2016. 2016.

KIYOSAKI, R.; LECHTER, S. Pai rico pai pobre. Rio de Janeiro: Campus, 2002.

LUCCI, C. R. et al. A influência da educação financeira nas decisões de consumo e investimento dos indivíduos. In: SEMINÁRIOS EM ADMINISTRAÇÃO DA UNIVERSIDADE DE SÃO PAULO, 9., 2006, São Paulo. Anais... São Paulo: USP, 2006.

LUSARDI, A.; SCHERESBERG, C. DE B. Financial literacy and high-cost borrowing in the United States. National Bureau of Economic Research, i. w18969, 2013.

MEIER, S.; SPRENGER, C. D. Discounting financial literacy: Time preferences and participation in financial education programs. Journal of Economic Behavior \& Organization, Estados Unidos, p. 159-174, 2012.

MESQUITA, P. S. B. Um modelo de Regressão Logística para Avaliação dos Programas de Pós-Graduação no Brasil. 2014. Dissertação (Mestrado)-Universidade Estadual do Norte Fluminense, Rio de Janeiro, 2014.

ORGANIZATION FOR ECONOMIC COOPERATION AND DEVELOPMENT. Annual Report. 2005.

ORGANIZATION FOR ECONOMIC COOPERATION AND DEVELOPMENT. International Survey of Adult Financial Literacy Competencies. 2016. 
PEREIRA, J.; LUCENA, W. G. L. A influência da educação financeira e os fatores emocionais: um estudo com alunos de Contabildiade e Engenharia. Revista de Administração e Negócios da Amazônia, Rondônia, v. 6, n. 3, 2014.

PEREIRA, M. T.; LOPES, J. L. A importância do capital humano para o crescimento econômico. In: ENCONTRO DE PRODUÇÃO CIENTÍFICA E TECNOLOGIA, 9., 2014, Campo Mourão. Anais... Campo Mourão, 2014.

RAMOS, B. de F.; MORAES JUNIOR, A. dos S. Educação financeira e mercaco de capitais: um estudo sobre a importância da desmitificação do mercado de capitais e educação financeira na sociedade brasileira. Revista Eletrônica de Debates em Economia, São Paulo, v. 1, n. 1, 2012.

RODRIGUES, A. C. A evolução do mercado de capitais e o perfil do acionista minoritário no Brasil. Scientia Iuris, Londrina, v. 16, n. 2, p. 107-128, 2012.

SCHULTZ, T. W. Investment in human capital. The American Economic Review, v. 51, i. 1, p. 1-17, 1961.

THOMAS, A.; SPARATO, L. Financial Literacy, Human Capital and Stock Market Participation in Europe: An Empirical Exercise under Endogenous Framework. Dipartimento di Economia e Management - Università di Pisa Discussion Paper, Gennaio, n. 194, 2015.

VAN ROOIJ, M.; LUSARDI, A.; ALESSI, R. Financial Literacy and Stock Market Participation. Journal of Economic Behavior \& Organization, Estados Unidos, v. 101, i. 2, p. 449-472, 2011.

VIANA, G.; LIMA, J. F. DE. Capital humano e crescimento econômico. Revista Internacional de Desenvolvimento Local, Campo Grande: Universidade Católica Dom Bosco, v. 11, n. 2, 2010.

WALTENBERG, F. D. Análise econômica de sistemas educativos: uma resenha crítica da literatura e avaliação empírica da iniqüidade do sistema educativo brasileiro. 2002. Dissertação (Mestrado)-Universidade de São Paulo, São Paulo, 2002.

WISNIEWSKI, M. L. G. A importância da educação financeira na gestão das finanças pessoais: uma ênfase na popularização do mercado de capitais brasileiro. Revista Intersaberes, Curitiba, ano 6, n. 12, p. 155-172, 2011. 
XIA, T.; WANG, Z.; LI, K. Financial Literacy Overconfidence and Stock Market Participation. Social Indicators Research, v. 119, i. 3, p. 1233-1245, 2014.

\title{
Como citar este artigo:
}

\begin{abstract}
ABNT
AMORIM, Klerton Andrade Freitas de et al. A influência da educação financeira na inserção dos investidores no mercado de capitais brasileiro: um estudo com discentes da área de negócios. RACE, Revista de Administração, Contabilidade e Economia, Joaçaba: Ed. Unoesc, v. 17, n. 2, p. 567-590, maio/ago. 2018. Disponível em: <http://editora.unoesc.edu.br/index.php/race>. Acesso em: dia/mês/ano.

APA

Amorim, K. A. F. de, Lucena, G. K. L., Pontes Girão, L. F. de A., \& Queiroz, D. B. de. (2018). A influência da educação financeira na inserção dos investidores no mercado de capitais brasileiro: um estudo com discentes da área de negócios. RACE, Revista de Administração, Contabilidade e Economia, 17(2), 567-590. Recuperado em dia/mês/ano, de http://editora.unoesc.edu.br/index.php/race
\end{abstract}

